\title{
Was ist Infektionskontrolle?
}

\author{
Roland Schulze-Röbbecke
}

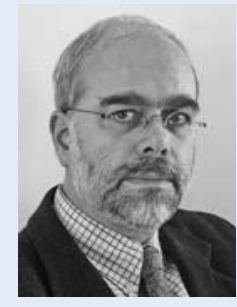

Roland SchulzeRöbbecke

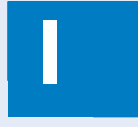

n deutschsprachigen Artikeln über Themen der Infektionsprävention und Krankenhaushygiene stößt man immer wieder auf das Wort „Kontrolle“, z. B. in Ausdrücken wie „Infektionskontrolle“ [1 - 3], „Kontrolle von MRSA“ [3] und „Kontrolle Katheter-assoziierter Harnwegsinfektionen“ [4]. Was ist mit diesem Rätselwort gemeint? Der Duden [5] kennt das Wort „Infektionskontrolle“ nicht und bietet für „Kontrolle“ folgende Bedeutungserklärungen an:

- dauernde Überwachung, Aufsicht, der jemand/etwas untersteht

- Überprüfung, der jemand/etwas unterzogen wird

- Herrschaft, Gewalt, die man über jemanden/sich/ etwas hat

Wie und mit welchem Ziel sollen also Infektionen und Erreger überwacht und überprüft oder soll Herrschaft über sie ausgeübt werden? Ziel ist offenbar der Infektionsschutz, die Infektionsprävention. Warum aber werden bereits im Titel einiger Arbeiten „Prävention“ und „Kontrolle“ gegenübergestellt $[3,4]$ ? Sollte es sich bei der Kontrolle etwa um Überwachungsmaßnahmen handeln, die die Prävention effektiv ergänzen?

Leser dieser Arbeiten erhalten keine Antwort auf solche Fragen.

Geht man der Sache auf den Grund so stellt sich heraus, dass „Infektionskontrolle“ die wörtliche Übersetzung des englischsprachigen Ausdrucks „infection control“ ist. Das Bedeutungsspektrum des englischen Wortes „control“ unterscheidet sich jedoch erheblich von dem des deutschen Wortes „Kontrolle“ und umfasst laut Oxford Dictionary [6] in erster Linie

- The power to influence or direct people's behaviour or the course of events und - im Zusammenhang mit unserem Thema

- The restriction of an activity, tendency, or phenomenon (e.g. crime control).
Entsprechend der zweiten Bedeutungserklärung bezeichnet der Begriff , infection control“ also die Eindämmung eines vorhandenen Infektionsgeschehens; in der englischen Fachsprache ist er weitgehend gleichbedeutend mit „prevention of the spread of infectious diseases" [7].

Versucht man den Begriff „infection control“ einer der klassischen Präventionskategorien zuzuordnen, nämlich der

- Primärprävention (Vermeidung der Entstehung einer Krankheit durch Beseitigung ihrer Ursachen),

- Sekundärprävention (Erkennung einer Krankheit im asymptomatischen Frühstadium) oder

- Tertiärprävention (Aufhalten der weiteren Verschlechterung oder Verminderung der Komplikationen einer bestehenden Krankheit) [8], so zählt er bezogen auf die Bevölkerung am ehesten zur Tertiärprävention (auch wenn er sich aus individualmedizinischer Sicht primär- und sekundärpräventiver Instrumente bedient, wie der Händedesinfektion zur Prävention der Erregerübertragung auf einen Patienten oder dem Screening von Einzelpersonen auf bestimmte Erreger). Im Englischen hat die Unterscheidung zwischen „infection control“ und ,infection prevention“ daher eine gewisse Berechtigung, wie auch im Falle der US-amerikanischen Bundesbehörde CDC, die ihren Namen Centers for Disease Control im Jahre 1992 in Centers for Disease Control and Prevention umänderte.

In letzter Zeit ist festzustellen, dass der Begriff ,infection control “ in der englischen Sprache einen Bedeutungswandel durchmacht und immer häufiger im Sinne von „prevention of healthcare-associated infections“ verstanden wird, wobei der Begriff „,healthcareassociated infection“ die unpräziseren Begriffe „hospital infection“ und „nosocomial infection“ heute immer mehr verdrängt [7,9]. Mit anderen Worten: Der Begriff „infection control“ unterliegt einerseits einer Bedeutungseinschränkung von der Gesamtheit aller Infektionen auf „nosokomiale Infektionen“ im Sinne der Legaldefinition in $\S 2$ des deutschen Infektionsschutz- 
gesetzes. Andererseits unterliegt er einer Bedeutungserweiterung von der Tertiärprävention auf alle Aspekte der Prävention.

Interessant ist in diesem Zusammenhang, dass die Präventivmedizin neben der Primär-, Sekundär- und Tertiärprävention inzwischen auch die Quartärprävention kennt $[10,11]$. Diese befasst sich mit der Identifizierung von Risiken der „Übermedikalisierung“, dem Schutz der Patienten vor überflüssigen medizinischen Maßnahmen und der Suche nach ethisch akzeptablen Alternativen. Im Bereich der Prävention nosokomialer Infektionen ist dieser Aspekt ebenfalls von Bedeutung, da Patienten durch Maßnahmen von unbewiesener Wirksamkeit und durch falsche Prioritätensetzung Schaden erleiden können [12].

Abschließend sei die Frage gestellt, ob das missverständliche Wort „Infektionskontrolle“ eine Bereicherung des deutschen Sprachschatzes darstellt. Nach Voltaire soll man nie ein neues Wort verwenden, sofern es nicht 3 Bedingungen erfüllt: Es muss notwendig sein, es muss verständlich sein und es muss wohlklingend sein. Ich meine, dass das Wort „Infektionskontrolle“ keine dieser Bedingungen erfüllt. Wichtiger als die Schaffung eines derartigen Neologismus, der mit den bereits vorhandenen Begriffen „Infektionsschutz“ und „Infektionsprävention“ konkurriert, wäre es im Bereich des Infektionsschutzes Anstrengungen zu unternehmen, in der schwammigen deutschsprachigen Terminologie mit Ausdrücken wie „unrein“, „Keimverschleppung“ und „Infektionshygiene“ allgemein für mehr Präzision und Klarheit zu sorgen. Wichtiger wäre es außerdem, auch die Quartärprävention zu einem wesentlichen Bestandteil der Infektionsprävention zu machen.

\section{Literatur}

1 Eigentler A, Heuberger S. Infektionskontrolle bei Meningokokkenerkrankungen. Wiener Medizinisches Magazin 2011; 14: $12-15$

2 Kramer A, Hübner NO, Steinmann J et al. Prävention und Ausbruchmanagement bei Auftreten von Norovirusinfektionen - Hygienemaßnahmen zur Infektionskontrolle im Krankenhaus. Klinikarzt 2009; 38: $182-186$

3 Kommission für Krankenhaushygiene und Infektionsprävention am RKI. Empfehlungen zur Prävention und Kontrolle von Methicillin-resistenten Staphylococcus aureus-Stämmen (MRSA) in Krankenhäusern und anderen medizinischen Einrichtungen. Bundesgesundheitsbl 1999; 42: 954-958

4 Kommission für Krankenhaushygiene und Infektionsprävention am Robert Koch-Institut. Empfehlungen zur Prävention und Kontrolle Katheterassoziierter Harnwegsinfektionen. Bundesgesundheitsbl 1999; 42: 806-809

5 Duden online. Im Internet: www.duden.de Stand: 14.1.2012

6 Oxford Dictionaries, Version „World English“. Im Internet: www.oxforddictionaries.com Stand: 14.1.2012

7 MedlinePlus, a service of the U.S.National Library of Medicine. Im Internet: www.nIm.nih.gov/ medlineplus

Stand: 14.01.2012

8 Fletcher RH, Fletcher SW. Clinical epidemiology: the essentials. 4th ed. Baltimore, Philadelphia: Lippincott Williams \& Wilkins; 2005

9 Wikipedia, The Free Encyclopedia. en.wikipedia. org/wiki/Infection_control Stand: 20.2.2012

10 Gofrit ON, Shemer J, Leibovici D et al. Quaternary prevention: a new look at an old challenge. Isr Med Assoc J. 2000; 2: 498-500

11 Starfield B, Hyde J, Gérvas J et al. The concept of prevention: a good idea gone astray? J Epidemiol Community Health 2008; 62: 580-583

12 Schulze-Röbbecke R. Primum nocere. Krankenhaushygiene up2date 2010; 5: 73-74 